www. revistadyo. com

\title{
Como a gamificação contribui para melhorar o desempenho da área de vendas?
}

Dr Elenise Angélica Martins da Rocha, Dr. Diego Augusto de Jesus Pacheco,

Dr. Giancarlo Medeiros Pereira

https://doi.org/10.37610/dyo.v0i75.608
Recibido: 23 de Noviembre de 2020

Aceptado: 8 de Octubre de 2021

\section{Resumo}

Este artigo analisa o uso da Gamificação no treinamento de vendedores de calçados ao longo de quatro anos, através de uma pesquisa-ação. A implementação do programa de treinamento aconteceu durante ciclos de implementação gerando melhorias e aprendizado para a empresa. De forma geral, os resultados da pesquisa possibilitaram minimizar problemas futuros de performance na a área de vendas. Os achados da pesquisa indicam que a Gamificação aprimorou o desempenho dos vendedores principalmente nas seguintes dimensões: argumentos de vendas, conhecimento dos produtos, e alternativas para o fechamento da venda. $\mathrm{O}$ processo de Gamificação implementado indicou oportunidades para se aprimorar o conteúdo dos treinamentos, a análise do processo de aprendizagem, e a percepção de realidade proporcionada pelos jogos. Esse estudo contribui indicando benefícios e os requisitos para a aplicação da gamificação no treinamento de vendedores. A escolha pela pesquisa-ação como metodologia e a utilização de ciclos de melhoria e aprendizagem aumentou a eficiência geral dos treinamentos, possibilitando a tomada de decisões mais rápidas, gerando melhores resultados e possibilitando replicações de boas decisões e correção de erros entre os ciclos.

\section{Palavras-chave}

Gamificação; Treinamento de Vendas; Análise de Aprendizagem, Simulador de Vendas; Games

\section{Introdução}

O treinamento e capacitação de vendedores é uma atividade estratégica para as organizações (Guenzi; Sajtos; Troilo, 2016). O treinamento contribui para ampliar a vantagem competitiva (Singh; Venugopal, 2015; Bachrach; Mullins; Rapp, 2017), alavancar o desempenho dos negócios (AllalChérif; Makhlouf, 2016; Bachrach; Mullins; Rapp, 2017), viabilizar a inovação em processos, produtos e serviços (Singh; Venugopal, 2015; Allal-Chérif; Makhlouf, 2016), e ampliar o uso das novas tecnologias (Tanner et al., 2008; Gordon et al., 2012; Guenzi; Sajtos; Troilo, 2016).

Dr Elenise Angélica Martins da Rocha * eleniser@unisinos.br

iD ORCID:0000-0002-2072-8395

Dr. Diego Augusto de Jesus Pacheco ** diego@btech.au.dk

iD ORCID:0000-0003-4453-7216

Dr Giancarlo Medeiros Pereira *** gian@unisinos.br

iD ORCID: 0000-0003-0539-2851

* University of Sinos Valley, Postgraduation Program in Production Engineering and Systems

Av. Unisinos 905, Brazil; Phone/Fax: +55 5135911122

** Aarhus University, School of Business and Social Scienc-

es (Aarhus BSS), Department of Business Development and Technology (BTECH) Birk Centerpark 157400 Herning, Denmark

*** Postgraduation Program in Production Engineering and Systems University of Sinos Valley Av. Unisinos 905, Brazil; Phone/Fax: +55 5135911122
Os treinamentos na área de vendas, todavia, precisam ir além do uso de recursos tecnológicos como videoaulas e mobile learning (aprendizagem através de dispositivos móveis) (Connolly et al., 2012), devendo, portanto, adotar novos recursos e metodologias. A gamificação é um exemplo desses novos recursos (Hamari; Koivisto, 2015; Groza; Locander; Howlett, 2016), ao proporcionar situações de competição e induzir a colaboração entre os participantes (De-Marcos et al., 2014). A competição é regulada pelos objetivos de desempenho pré-definidos (Mačiuliene; Skaržauskiene, 2016), de forma a incrementar o aprendizado dos participantes (Buckley; Doyle, 2017). Se por um lado os jogos sérios de uma maneira geral podem ser considerados jogos com propósitos de não-entretenimento que utilizam mudanças educacionais e comportamentais para o uso (De Freitas; Ketelhut, 2014), a estratégia de gamificação pretende mudar o comportamento e competências a partir do engajamento significativo sujeito a recompensas (Carvalho et al., 2015). Tais elementos têm ampliado a adoção da gamificação no ambiente empresarial (Hamari; Koivisto, 2015; Attali; Arieli-Attali, 2015; Hamari; Keronen, 2017; $\mathrm{Xu}$; Buhalis; Weber, 2017) e acadêmico (De Freitas; Ketelhut, 2014).

Os games estão sendo utilizados para selecionar, integrar e treinar funcionários (Allal-Chérif; Makhlouf, 2016), viabilizar uma imersão rápida em algum cenário (Domínguez et al., 2013; Raybourn, 2014; Westera, 2017), incrementar a atratividade de conteúdos (Raybourn, 2014), (AllalChérif; Makhlouf, 2016), alinhar a formação aos objetivos do negócio (Singh; Venugopal, 2015, engajar as gerações digitais (Buckley; Doyle, 2017) e simular cenários (Howard, 2017). O uso de games no treinamento de vendedores, em 
particular, alavanca vendas (Bashaw et al., 2002), melhora o entendimento acerca dos produtos e processos (Samaddar; Kadiyala, 2006), aumenta a participação dos profissionais geograficamente dispersos (Li; Jhang-Li, 2010), aprimora a capacitação (Qudrat-Ullah, 2010; Gordon et al., 2012 (Hamari; Koivisto, 2015) e facilita a Gestão do Conhecimento (Allal-Chérif; Makhlouf, 2016).

No que diz respeito à importância do tema, a literatura ainda é reduzida e carece de estudos acerca das variaveis envolvendo a implementação da Gamificação no treinamento de vendedores. Em especial, destaca-se a necessidade de se aprimorar o entendimento sobre como a gamificação aplicada ao treinamento de vendedores pode gerar melhores resultados para o negócio (Raybourn, 2014), quais são as melhores práticas para o treinamento desses profissionais (Li; Jhang-Li, 2010; Raybourn, 2014), e quais treinamentos mais se adequam ao uso da gamificação (Raybourn, 2014; Allal-Chérif; Makhlouf, 2016). Portanto, a análise dessas lacunas de conhecimento levou a elaboração da seguinte questão de pesquisa:

RQ. Como a estratëgia de gamificação contribui para melhorar o desempenho da área de vendas?

A investigação da questão de pesquisa desse estudo foi conduzida na empresa do setor calçadista Sapatus (nome fictício), através da análise por quatro anos de um projeto de gamificação. Ao longo dos quatro anos, a Sapatus já utilizou a gamificação para capacitar 17,652 profissionais da área comercial. $\mathrm{O}$ artigo foi estruturado da seguinte forma: após esta introdução, a próxima seção apresenta a revisão da literatura sobre treinamento em vendas que, aborda um conjunto de temas relevantes para a elaboração desta pesquisa, visando delinear o conteúdo teórico para embasar o estudo proposto por este artigo. Em seguida, a seção de metodologia apresenta o método de pesquisa, coleta e análise de dados, além de detalhas sua implementação. A seção de Descobertas trata da discussão do estudo e, na Seção de Conclusões, oferece as conclusões finais.

\section{Treinamento em Vendas}

O uso tecnologias vem ganhando espaço na formação da força de vendas (Moncrief; Marshall; Rudd, 2015; Yalabik; Howard; Roden, 2012), tem sido destacado pela sua contribuição em realizas a entrega eficiente de materiais (Halkett, 2002), promover o engajamento das pessoas (Honeycutt; Karande; Jantan, 2002), e permitir a captação do conhecimento explícito e tácito dos vendedores (Tanner et al., 2008). A tecnologia também permite acompanhar e avaliar a aprendizagem. Isso abarca o controle sobre o conteúdo assimilado, o tempo necessário para a assimilação, e a identificação de problemas no aprendizado (Honeycutt; Karande; Jantan, 2002).
A literatura indica que a tecnologia deve ser acompanhada de uma metodologia dinâmica e envolvente, de formas a viabilizar os objetivos de formação almejados para o público alvo (Harman; Koohang; Paliszkiewicz, 2014), e dentre as opções disponíveis destaca-se como tendência o uso da gamificação (Groza; Locander; Howlett, 2016; Mačiuliene; Skaržauskiene, 2016; Serna; Bachiller; Serna, 2017), principalmente nos setores comerciais (Yalabik; Howard; Roden, 2012; Lauto; Valentin, 2016; Xu; Buhalis; Weber, 2017). Em comparação aos treinamentos convencionais, o uso de tecnologias em treinamentos de vendas contribui para reduzir o tempo de treinamento em até $40 \%$, e reduz os custos em até 50\% (Honeycutt; Karande; Jantan, 2002). No sentido de identificar os conceitos e os principais processos para a aplicação da gamificação, a próxima seção apresenta os principais pressupostos teóricos encontrados na literatura sobre gamificação em treinamento de vendas.

\subsection{Gamificação em Treinamento de Vendas}

Apesar do número crescente de estudos que demonstraram interesse no tema da gamificação (Harman; Koohang; Paliszkiewicz, 2014; Malegiannaki; Daradoumis, 2017), na literatura pesquisada, não se evidencia uma única definição que seja cientificamente e amplamente aceita (Xu; Buhalis; Weber, 2017). Dessa forma, para efeitos deste estudo, a gamificação é definida como uma estratégia que utiliza elementos que se baseiam na Teoria dos Jogos para aplicação em um contexto de não-jogo (Deterding et al., 2011). Como tal, a utilização de uma estratégia gamificada não envolve necessariamente o uso de um jogo ou o emprego de tecnologias digitais (Buckley; Doyle, 2017). A Teoria dos Jogos relaciona-se ao estudo de como as pessoas, empresas ou nações (agentes ou jogadores, na linguagem dos jogos) determinam estratégias em situações diferentes quando enfrentam estratégias concorrentes de outros agentes. Este aspecto da Teoria dos Jogos é o que motiva seu uso como uma ferramenta para gerar mudanças de comportamentos e reflexões sobre a aprendizagem (Benito-Ostolaza; SanchisLlopis, 2014).

As diferenças conceituais entre gamificação, jogos e os jogos sérios não são apresentadas com muita clareza na literatura pesquisada, embora alguns autores tenham se preocupado em distingui-los. Enquanto que os jogos sérios em geral são descritos como jogos para propósitos de nãoentretenimento e que, muitas vezes, utilizam mudanças educacionais e comportamentais como motores-chave para o uso (De Freitas; Ketelhut, 2014), a gamificação se concentra na estratégia para mudar o comportamento dos jogadores ou resolver problemas, envolvendo-os com o seu ambiente e com outros jogadores para promover uma interação e um engajamento significativo que potencialmente está sujeito a obter recompensas (Carvalho et al., 2015; Ghanbari; Simila; Markkula, 2015). Em contrapartida, o jogo se concentra no entretenimento e no prazer da experiência (De Freitas; 
Ketelhut, 2014), podendo ser definido a partir de um subconjunto da gamificação (Kapp, 2012).

Estudos sobre os benefícios potenciais advindos do uso da gamificação em programas de formação mostram a contribuição para: registrar e acompanhar os estágios iniciais e de desenvolvimento do usuário (Serrano-Laguna et al., 2017; Westera, 2017), investigar profundamente as variáveis e tomadas de decisões (Singh; Venugopal, 2015; SerranoLaguna et al., 2017; Westera, 2017), permitir feedback imediato, raciocínio cognitivo e retenção de informações (Gordon et al., 2012; Yalabik; Howard; Roden, 2012), gerar mudanças de comportamento explícitas, implícitas ou uma combinação de ambas (De Freitas; Ketelhut, 2014; Ghanbari; Simila; Markkula, 2015; Buckley; Doyle, 2017), promover aprendizagem imersiva e envolvente (Yalabik; Howard; Roden, 2012; Westera, 2017), promover a qualidade dos programas de aprendizagem, simular cenários adaptados para a formação de pessoas na organização (Qudrat-Ullah, 2010; Gordon et al., 2012; Howard, 2017) mesmo frente às múltiplas geografias e culturas de diferentes realidades (Ballance, 2013), escalar rapidamente a imersão no treinamento (Domínguez et al., 2013; Raybourn, 2014; Westera, 2017), alinhar as estratégias de formação de vendedores aos objetivos organizacionais na área de vendas (Moncrief; Marshall; Rudd, 2015; Singh; Venugopal, 2015), engajar gerações digitais (Moncrief; Marshall; Rudd, 2015; Buckley; Doyle, 2017), adicionar elementos motivacionais ao formato de entrega dos conteúdos (Domínguez et al., 2013; Jung; Kim; Lee, 2014; Mačiulienė; Skaržauskienė, 2016), criar ambientes controlados para testar a capacidade dos jogadores em análise de situações (Yalabik; Howard; Roden, 2012), tomada de decisões (Bevilacqua et al., 2015), administração de imprevistos e eventos inesperados, otimização de recursos e coordenação de habilidades (Singh; Venugopal, 2015; Howard, 2017; Westera, 2017), concentrar esforços da área comercial em diagnóstico e treinamento (Singh; Venugopal, 2015; Allal-Chérif; Makhlouf, 2016), aprimorar o desempenho profissional (Jung; Kim; Lee, 2014; Singh; Venugopal, 2015; Allal-Chérif; Makhlouf, 2016), resolver problemas em uma ampla gama de áreas (Harman; Koohang; Paliszkiewicz, 2014), criar um fluxo cognitivo entre as capacidades do jogador e os desafios oferecidos (Westera, 2017), analisar e medir os resultados de aprendizagem dos jogadores (Serrano-Laguna et al., 2017), solucionar problemas motivacionais dentro de contextos de aprendizagem ou de trabalho (Sailer et al., 2017) e descrever as interações do jogador, navegação e história de atividades e conquistas (Serrano-Laguna et al., 2017; Westera, 2017).

A gamificação pode representar um avanço na realização de experiências de aprendizagem participativas, motivadoras, envolventes e significativas (De Marcos et al., 2014), sendo vista como uma ferramenta para apoiar a estratégia de negócio que oportuniza pensar a aplicação prática em diferentes áreas e contextos: concepção de produtos serviços e sistemas (Yalabik; Howard; Roden, 2012), treinamento técnico, treinamento de liderança, treinamento de vendas, treinamento de produtos, avaliação de desempenho e avaliação da produtividade (Ballance, 2013), treinamento estratégico (Benito-Ostolaza; Sanchis-Llopis, 2014), inovação de produto (Jung; Kim; Lee, 2014; Lauto; Valentin, 2016), mudança e design cognitivo (Ghanbari; Simila; Markkula, 2015), gestão do conhecimento (Ghanbari; Simila; Markkula, 2015; Allal-Chérif; Makhlouf, 2016), capacidade de gestão de sistemas (Arslan; Yuksel; Gunes, 2015) e avaliação do ambiente organizacional e de mercado (Yalabik; Howard; Roden, 2012; Bevilacqua, et al., 2015).

Os resultados advindos da aplicação da gamificação no contexto de formação da área de vendas mostraram a sua contribuição para, aumentar os níveis de conhecimentos dos vendedores, reduzir o ciclo de formação, aumentar suas habilidades e aprimorar a capacidade de vender (Bashaw; Ingram; Keillor, 2002), criar um sistema de colaboração que resultaram no desenvolvimento de novos produtos e obtenção de melhorias em produtos e processos existentes (Samaddar; Kadiyala, 2006), aumentar o envolvimento dos funcionários geograficamente dispersos (Li; Jhang-Li, 2010), aumentar a participação nas avaliações de desempenho (Rapp et al., 2012), desenvolver competências e promover a motivação da força de vendas (Kananen, 2013), melhorar a captação de requisitos do consumidor para segmentação dos clientes (Jung; Kim; Lee, 2014), aumentar o desempenho na formação (Rodriguez; Honeycutt; Ragland, 2015), aumentar a aderência da nova geração digital de vendedores nos treinamentos (Moncrief; Marshall; Rudd, 2015), criar um sistema de recompensas que, em termos de motivação, aumenta a produtividade dos funcionários (Little, 2015), aumentar recebimento virtual de propostas para potenciais novos produtos em troca de recompensas virtuais pelas melhores ideias (Lauto; Valentin, 2016), melhorar a Gestão do Conhecimento (Allal-Chérif; Makhlouf, 2016) e, por fim, aumentar a notoriedade da marca, nível de satisfação e lealdade dos clientes (Xu; Buhalis; Weber, 2017).

Da mesma forma que o interesse pela gamificação vem crescendo, poucos estudos têm demonstrado seus efeitos negativos (Seaborn; Fels, 2015; Andrade; Mizoguchi; Isotani, 2016). Algumas barreiras poderão ser evidenciadas nas competições embasadas em incentivos, já que é necessário o acompanhamento e avaliação dos impactos, riscos e das implicações éticas ou legais observadas nos comportamentos gerados (Murphy; Dacin; Ford, 2004) e nas atitudes dos vendedores para participar e perseguir objetivos (Murphy; Dacin, 2009). Estudos sobre o uso da gamificação para estimular a partilha de conhecimento mostraram que a recompensa baseada em grupos não é apenas menos eficiente do que a recompensa individual, mas também sujeita a um problema potencial de produtividade, no qual os trabalhadores mais produtivos podem não participar na partilha de conhecimento (Lee; Ahn, 2007). As organizações precisam entender que os benefícios materiais e prêmios extrínsecos devem ser escolhidos cuidadosamente para reconhecer o compartilhamento de conhecimento, uma vez que nem sempre podem automaticamente dar retornos 
positivos. Recompensas intrínsecas através de gratificação virtual e reconhecimento social podem ser uma alternativa mais adequada para motivar as pessoas (Pandey; Dutta, 2013).

Com relação a aplicabilidade da Gamificação, uma concepção educacional cuidadosa conduzida por objetivos claros é essencial para uma integração significativa da gamificação nas abordagens de aprendizagem (Connolly et al., 2012). Os efeitos da gamificação dependem muito do contexto em que ela é implementada e de seus usuários (De Marcos et al., 2014). Para aplicar jogos na aprendizagem, é importante ter uma compreensão das tarefas, atividades, habilidades e operações que diferentes tipos de jogos podem oferecer, além disso, examinar como estes podem corresponder aos desejados resultados de aprendizagem (Connolly et al., 2012). Programas de treinamento com o uso de tecnologias na área de vendas precisam prever também apoio e assistência tecnológica para eliminar algumas barreiras como a apreensão de alguns profissionais para o uso da tecnologia (Robinson; Marshall; Stamps, 2005; Hamari; Keronen, 2017) e observar ainda que, conforme o estilo de aprendizagem de um indivíduo, é preciso definir a forma apropriada de uma intervenção instrucional, respeitando o impacto sobre a sua obtenção de resultados de aprendizagem (Buckley; Doyle, 2017). O desenvolvimento de jogos para a aprendizagem pode ser muito complexo e dispendioso (Boyle et al., 2016), pois as próprias características dos jogadores também podem alterar a eficácia da aprendizagem, considerando, por exemplo, as suas motivações e/ou deficiência de conhecimento prévio necessário para realizar uma tarefa (Westera, 2017).

\subsection{Análise da Aprendizagem através do uso da Gamificação}

Learning Analytics ou análise da aprendizagem, é um campo emergente focado na análise das interações dos usuários com conteúdo educacional (Serrano-Laguna et al., 2017). A análise das interações dos usuários fornece evidências para previsões dos comportamentos futuros dos usuários (Ifenthaler; Widanapathirana, 2014). A natureza interativa da gamificação e dos games simuladores faz deles uma boa fonte de dados para análise da aprendizagem, permitindo acesso ao feedback instantâneo para avaliar e refletir sobre o desempenho alcançado (De-Marcos; GarciaLopez; Garcia-Cabot, 2016; Howard, 2017; De-Marcos et al., 2017). Essa experiência interativa aumenta a qualidade da aquisição das competências pelos usuários, uma vez que estes sistemas oferecem uma boa adaptabilidade às necessidades das pessoas e ao estilo de aprendizagem. Ou seja, a personalização contribui para aumentar a motivação dos usuários durante o treinamento (Borsci; Lawson; Broome, 2015). A possibilidade de acompanhar a sequência de ações realizadas pelos usuários em um contexto específico é representada por uma "atividade", cujos principais atributos são um ator (que executa a ação), um verbo (que ação é realizada) e um objeto (o alvo da ação). Uma sequência de atividades é chamada de "fluxo" que representa as ações de um conjunto de usuários (Serrano-Laguna et al., 2017).

As simulações podem contribuir para gerar informações sobre os usuários, para determinar:padrões de comportamento, padrões de pensamento, execução de processos e definições de prioridades e interesses. Contudo, para simular situações reais, é preciso planejar o alcance de determinados objetivos de aprendizagem (Xu; Buhalis; Weber, 2017). O uso de simuladores fornece oportunidade para os tomadores de decisões e aos próprios usuários testarem suas estratégias de decisão antes que a implementação de ações na vida real seja dispendiosa e muitas vezes irreversível (Tang; Austin, 2009). Cabe refletir que poucos estudos empíricos têm buscado identificar o valor da capacidade de absorção do conhecimento, que está diretamente relacionado a noções de aprendizagem organizacional (Murovec; Prodan, 2009).

No entanto, com relação ao desempenho da força de vendas, cinco diretrizes foram definidas por Robson et al. (2015) com o objetivo de resumir um conjunto de orientações para ajudar as empresas a incrementar resultados usando a gamificação. São elas: (i) definir a escolha das mecânicas e dinâmicas de forma que estejam orientadas por metas (financeiras, sociais ou ambientais) e com o objetivo de produzir ou ajustar comportamentos e resultados necessários para atingir os objetivos de negócio; (ii) compreender em profundidade as ligações entre as mecânicas, dinâmicas, emoções e os resultados relativos à aplicação da gamificação; (iii) compreender os aspectos positivos e negativos da aplicação da gamificação; (iv) monitorar constantemente a experiência de uso da gamificação e realizar ajustes necessários durante o processo de aplicação, analisando se ainda faz sentido e se o interesse do público alvo se mantém; e (v) concluir o processo garantindo que os usuários se disponham a voltar e se envolver com novos processos gamificados (Robson et al., 2015). Na próxima seção, serão apresentados os procedimentos metodológicos que nortearam esta pesquisa.

\section{Método de Pesquisa}

Esse estudo examinou como as empresas podem aprimorar o treinamento de seus vendedores por meio da adoção da gamificação, analisando um projeto de gamificação durante quatro anos em uma empresa do setor calçadista. Tal projeto teve a participação direta do pesquisador em colaborar internamente na empresa analisada, conduzir o grupo de trabalho dentro da empresa, mobilizar os participantes do grupo de trabalho ao longo dos quatro ciclos de pesquisaação conduzido, e por fim assessorando a empresa a construir e implantar os novos conhecimentos e competências geradas ao longo do processo de implantação da pesquisa ação. Nesse sentido, a pesquisa-ação um método de pesquisa adequado, pois analisa a contingência de um problema, com objetivo de abranger e reunir conhecimentos necessários para avaliar 
a solução. De acordo com Thiollent (2011), a pesquisa-ação é uma pesquisa social com base empírica, a qual envolve os pesquisadores e participantes da situação ou problema de forma cooperativa ou participativa. Tal método de pesquisa provou ser eficiente em problemas analisados através da aplicação da gamificação, de forma que, permitiu monitorar constantemente a experiência de uso da gamificação na empresa e realizar ajustes necessários durante o processo de aplicação, garantindo que os usuários se dispusessem a voltar e se envolver com novos processos gamificados (Robson et al., 2015).

Segundo Westbrook (1995), não há padrão a ser seguido para aplicar a pesquisa-ação. No entanto, existem alguns passos básicos de condução. Para realizar um estudo de pesquisa-ação, Mello et al. (2012) propõem um modelo adaptado de Westbrook (1995) e Coughlan e Coghlan (2002).
Nesse modelo (Figura 1), existem cinco fases em cada ciclo, as quais foram adotadas no presente estudo: Planejamento de Pesquisa-Ação (1), Coleta de Dados (2), Análise de Dados e Planejamento de Ações (3), Implementação (4) e Análise de Resultados (5). As fases de planejamento são compostas por duas etapas: seleção da unidade a ser estudada e a equipe de pesquisa-ação. Além disso, uma das principais premissas deste estudo é que cada uma das cinco fases pode ocorrer em ciclos de melhoria e aprendizagem, que visam avaliar os resultados obtidos e preparar uma base de conhecimento para novos ciclos de (re) planejamento. Paes et al. (2016) e Fagundes et al. (2017), argumentam que o uso de ciclos de melhoria e aprendizado foi um dos principais contribuintes para o sucesso da pesquisa-ação. O ciclo é composto por quatro etapas, Planejamento (P), Implementação (I), Observação e Avaliação (O \& A) e Reflexão e Ação (R \& A).
Figura 1 Ciclo da pesquisa-

ação.

Fonte: adaptado de Mello et al. (2012)

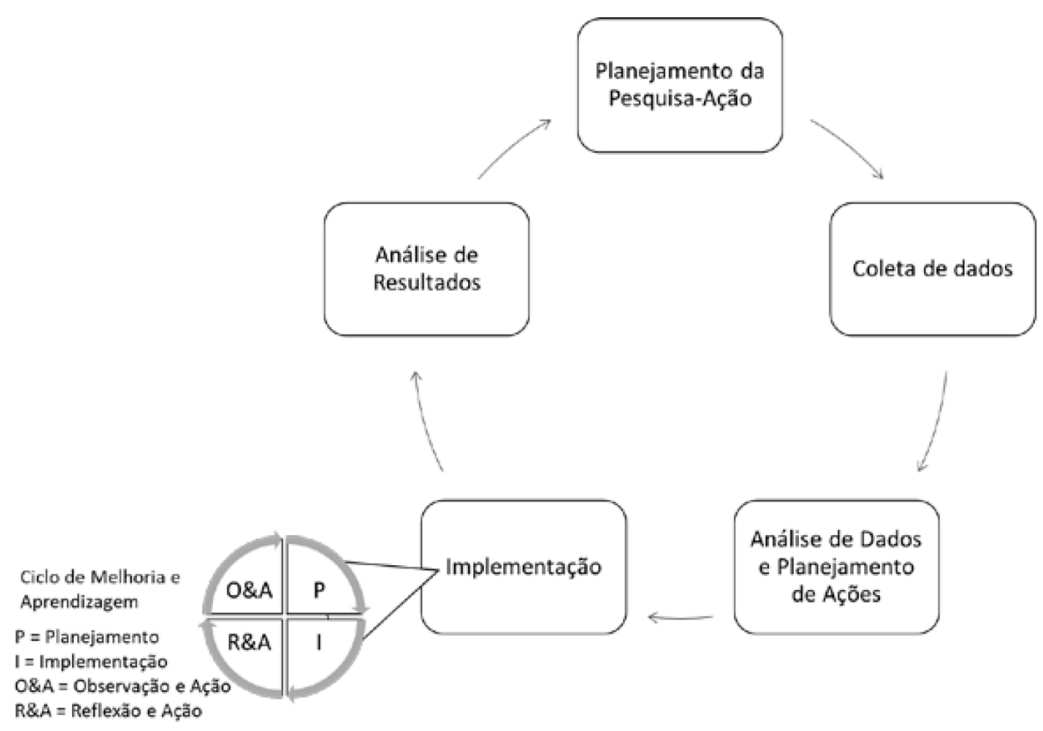

\subsection{Planejamento}

Assim, na fase de implementação, ocorreram quatro ciclos de melhoria e aprendizado durante a realização da pesquisa. Vale ressaltar que, durante a etapa de Reflexão e Ação (R \& A), foram realizadas análises dos principais desafios verificados em cada ciclo, bem como a identificação e execução de ações para resolver essas dificuldades. Para isso, foi utilizado como diretriz a literatura atual sobre Treinamento em Vendas e Gamificação. As seções a seguir apresentam as fases de implementação da Gamificação no Treinamento de Vendas por meio da pesquisa-ação conduzida, detalhando cada uma das etapas distintas: Planejamento de PesquisaAção; Coleta de dados; Análise de Dados e Planejamento de Ações; Implementação através de Ciclo de Melhoria e Aprendizagem e Análise de Resultados.
O planejamento da pesquisa-ação envolveu duas fases principais: seleção da unidade em estudo e definição da equipe. Neste estudo, o critério estabelecido para a seleção da unidade de pesquisa foi encontrar uma empresa que ainda não possuía um programa de treinamento de vendas com o uso da gamificação, ou que ainda estivesse na fase inicial de implementação deste programa. Portanto, à medida que esta pesquisa procurou explorar um tema que se encontra em avanço significativo na fronteira do conhecimento, poucos casos empíricos foram encontrados para serem analisados. A seleção do caso foi baseada em diretrizes da literatura (Barratt; Choi; Li, 2011) e por conveniência e acesso a empresa a partir da rede de contato dos pesquisadores. Um 
dos pesquisadores liderou um projeto de implementação da gamificação na área de vendas na empresa Sapatus. Portanto, a utilização deste caso objetivou conferir abrangência à pesquisa e validade às conclusões alcançadas.

A unidade de estudo investigada foi o treinamento de vendas com uso da Gamificação da empresa Sapatus (um pseudônimo). Trata-se de um fabricante de calçados situado no Brasil que possui mais de 70 anos e 18000 funcionários. A empresa possui faturamento em torno de 550 milhões de Dólares (aproximadamente 450 milhões de Euros), possui 246 lojas - entre próprias e franqueadas, oito unidades industriais - e está presente em 18 países, onde também produz sob encomenda de grandes marcas globais. Considerando a receita total, 180 milhões de Dólares (aproximadamente 145 milhões de Euros) vêm das vendas do varejo e o restante da receita vem da indústria e das marcas próprias. A Sapatus é considerada no mercado calçadista nacional como sendo uma empresa especialista em calçados que atua de forma única no segmento, pois possui domínio sobre a cadeia produtiva completa e a legítima propriedade em todas as dimensões do seu negócio, da indústria ao varejo.

O estudo em questão, exigiu profundo envolvimento dos pesquisadores durante os ciclos de implementação devido a amplitude e complexidade do assunto sob investigação. $\mathrm{O}$ que resultou que os treinamentos que o pesquisador líder realizou para os funcionários da empresa, precisaram ser acompanhados do início ao final para avaliar os resultados alcançados. Os gestores representantes da área de Educação Corporativa e responsáveis pela área de treinamentos de vendas da empresa apoiaram o planejamento da pesquisaação. Portanto, foram incluídos e definidos como membros da equipe do projeto de pesquisa-ação junto com o pesquisador líder. A Tabela 1 apresenta a equipe de pesquisa-ação, sua experiência como gestores, a codificação das reuniões e sua duração após cada ciclo encerrado.

Tabla 1 Perfil dos

entrevistados

\begin{tabular}{|l|l|l|}
\hline Gerente & Experiência & Duração \\
\hline A. Gerente do Varejo & 15 years & $\begin{array}{l}\text { Aproximadamente 60 } \\
\text { min }\end{array}$ \\
\hline B. Gerente de Marcas Próprias & 16 years & $\begin{array}{l}\text { Aproximadamente 60 } \\
\text { min }\end{array}$ \\
\hline C. Gerente Corporativo & 15 years & $\begin{array}{l}\text { Aproximadamente 60 } \\
\text { min }\end{array}$ \\
\hline D. Diretora de RH & 20 years & $\begin{array}{l}\text { Aproximadamente 60 } \\
\text { min }\end{array}$ \\
\hline
\end{tabular}

No presente estudo, foram realizadas reuniões ao final de cada ciclo para avaliar os resultados auferidos. Com base na literatura pesquisada as evidências coletadas, foram analisadas incluindo os resultados das reuniões e documentos fornecidos pela empresa para consulta.

\subsection{Coleta de dados}

Durante a coleta de dados observou-se que, historicamente os treinamentos de vendas ocorriam com baixa adesão dos vendedores e das lojas. Em média apenas 20\% dos vendedores e $20 \%$ das lojas participavam dos treinamentos de vendas. A lojas avaliavam os treinamentos como, em média, 70\% satisfatórios. Com base nessa análise, a conclusão dos pesquisadores foi de que não havia aderência dos vendedores aos treinamentos de vendas. O próximo passo foi o planejamento das ações subsequentes com base em algumas questões-chave:

- Quais ações poderiam motivar a participação dos vendedores em treinamentos? A empresa resolveu adotar a gamificação para tornar mais lúdico e atrativo os treinamentos para os vendedores.
- Qual é a melhor forma de levar o conteúdo até as lojas? A empresa resolveu investir em treinamentos online para que o vendedor escolhesse quando e onde estudar conforme sua disponibilidade. Posterior ao treinamento online, o simulador tem o objetivo de simular e avaliar as decisões dos vendedores, identificando o quanto houve apropriação do conteúdo.

- Qual é a forma de apresentação do conteúdo contribui mais para a aprendizagem dos vendedores? A empresa resolveu investir em simuladores virtuais que retratam ações do dia a dia do vendedor. Após a aplicação do simulador serão identificadas informações como: tomada de decisão correta e incorreta por treinando, unidade de negócio e processos; \% ações diante de restrições e oportunidades; \% das escolhas corretas e incorretas por treinando, unidade de negócio e processo; Nível de Prontidão da força de vendas; e Predição e mitigação de problemas futuros.

- Como produzir o conteúdo dos treinamentos dos vendedores? A empresa decidiu produzir internamente os conteúdos dos vendedores para que estivessem alinhados e contextualizados com a realidade. O simulador 
foi construído por uma consultoria de tecnologias educacionais a partir da entrega do conteúdo elaborado pela empresa.
Exemplo de uma das telas de interface do simulador é apresentado na Figura 2. A figura apresenta a imagem da interface do módulo de Formação de Sistema de Operação de Caixa do game simulador.
Figura 2 Interface do módulo de Formação de Sistema de Operação de Caixa do game simulador. Fonte: SCORM do LMS da Sapatus

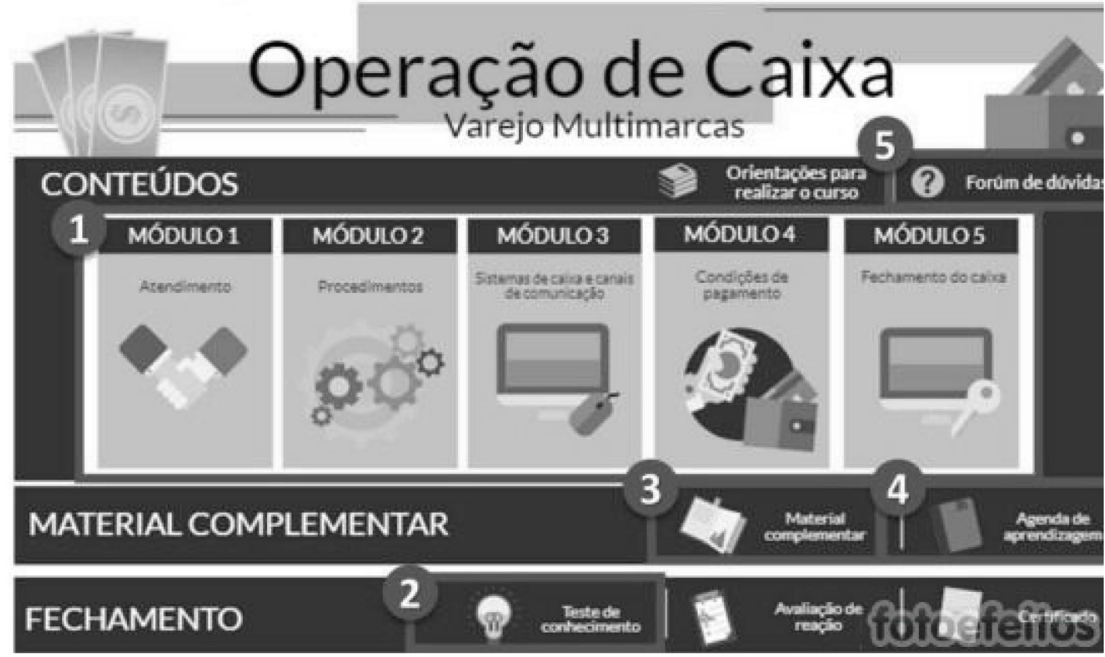

A construção da capacitação sobre o Sistema de Operação de Caixa foi desenhada a partir de cinco elementos fundamentais para que a empresa pudesse utilizar a análise prescritiva a partir dos resultados da aprendizagem ao identificar possíveis falhas. Com relação ao item (1) destacada em vermelho 'Conteúdos', cada um dos módulos entregou conteúdos no formato de simuladores, que contextualizaram desde o início do atendimento de um cliente até a finalização da compra. Todos os cinco simuladores permitiam que o vendedor acessasse conteúdos explicativos e, após adquiri-los, testasse a aplicação do seu conhecimento tomando decisões num contexto controlado e simulado, muito semelhante ao seu dia a dia de loja. Sobre o item (2) 'Teste de conhecimentos', é empregado como um instrumento de avaliação, assim como o simulador também exerce esse papel. A diferença é que o game simulador, ao mesmo tempo em que entrega conteúdos, propõe atividades avaliativas, e o vendedor não evolui no simulador se não tomar a decisão correta. No teste, ele pode evoluir mesmo errando uma questão e terá acesso a sua nota e feedback sobre acertos e erros apenas no final. O teste se apresenta em formato de Quiz, por meio de perguntas objetivas, trazendo situações contextualizadas e problemas reais de loja para avaliar a aprendizagem do vendedor e reforçar conceitos e práticas fundamentais. Sobre o item (3) 'Material complementar', a empresa recomenda, através de uma lista, materiais complementares, procedimentos, dicas práticas, materiais de leitura e informações que possam contribuir para ampliar o conhecimento do vendedor. E, na medida em que o vendedor possa necessitar ou vir a desejar reforçar alguns dos conhecimentos apresentados na formação, ele tem livre acesso aos materiais complementares. Outro recurso importante é a “Agenda de Aprendizagem”, item (4). Trata-se de uma página de feedback sobre os resultados de aprendizagem individual de cada vendedor. Disponibilizada com o objetivo de mostrar o resultado geral da aprendizagem de cada vendedor, nesta página, são apresentados os pontos fortes, neutros e fracos em uma lista personalizada de recomendações automáticas. As recomendações objetivavam reforçar a aprendizagem e eliminar os pontos fracos, bem como recomendar o aprofundamento em temas do curso e de outros cursos de interesse para cada vendedor. Os gráficos mostram o desempenho individual do vendedor no curso e por conteúdo. Adicionalmente, a empresa começou a implementar um recurso chamado de "Fórum de dúvidas" (5). Trata-se de um espaço para postagem de dúvidas, criado em formato de fórum. As dúvidas relacionadas ao curso são postadas pelos vendedores e um especialista responsável pelo tema da formação fica disponível para receber por e-mail essas dúvidas e, com certa brevidade, responder as publicações.

\subsection{Análise de dados e planejamento de ações}

A empresa realiza o lançamento das novas coleções anualmente através de treinamento online para toda a sua cadeia comercial. Esses treinamentos são responsáveis por apresentar informações sobre os novos produtos e argumentos de venda, conforme o perfil de clientes. Os vendedores possuem acesso ao ambiente virtual de aprendizagem da empresa através de um computador em loja, adquirido para fins específicos de treinamentos. Além disso, o vendedor pode acessar o ambiente virtual fora da empresa, e até mesmo através de dispositivos móveis. Cada gerente de loja, é responsável por criar mecanismos que estimulem e oportunizem que seus vendedores estudem durante o horário de trabalho. 
O treinamento online ocorre dois meses antes da nova coleção de calçados chegar nas lojas. Após o treinamento online já ter sido realizado, cada vendedor tem acesso ao simulador de vendas. O simulador retrata situações reais de atendimento, passando por todas as principais etapas da venda: abordagem, sondagem, contorno de objeções e fechamento da venda. Ao passar o mouse sobre o cliente, o sistema mostra o seu perfil (primeira informação que precisa ser interpretada pelo vendedor).

Após ler as informações do perfil do cliente, ele começa a executar as etapas da venda. $\mathrm{Na}$ etapa da abordagem o vendedor precisa receber o cliente na loja, escolhendo o diálogo dentre as opções oferecidas no simulador. O sistema vai direcionando o vendedor para diferentes situações em que ele terá que realizar escolhas na condução do processo de venda.
Através do desempenho individual no simulador, a sequência de ações realizadas pelos vendedores em diferentes contextos é representada por suas decisões. O fluxo de decisões de cada vendedor registrado no sistema, permite uma avaliação e identificação do seu nível de prontidão para agir em situações do dia a dia de loja. Após a fase de planejamento, em seguida veio a implementação da ação.

\subsection{Implementação de ação}

Cada ciclo da pesquisa-ação durou o período de 30 (trinta) dias. No decorrer de 4 (quatro) anos ocorreram 4 (quatro) ciclos por causa do acompanhamento dos dados dos games oriundos do lançamento anual das novas coleções. A fase de implementação, foi realizada com quatro ciclos de melhorias e aprendizados conforme Figura 3.
Figura 3 Implementação dos quatro ciclos de melhorias e aprendizados

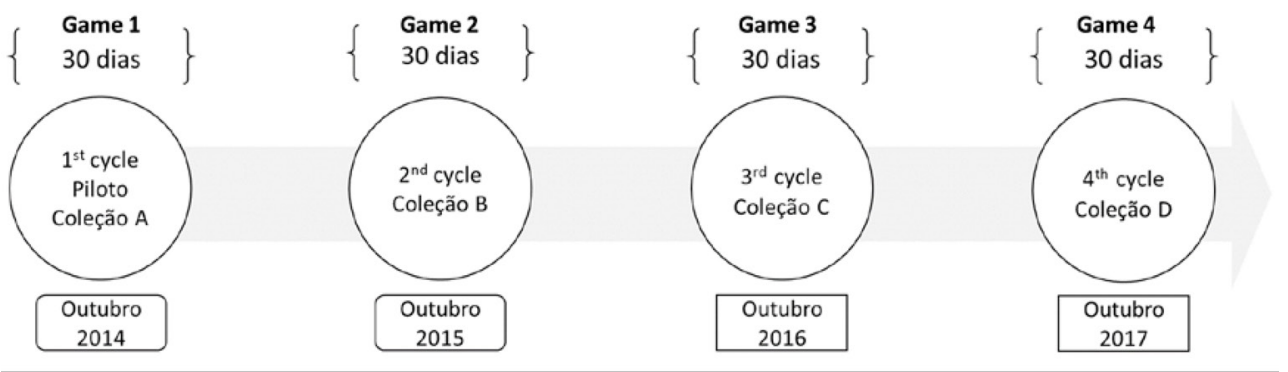

Quanto a estrutura do simulador, os vendedores reclamaram que o game era dispendioso demais tanto pelo volume quanto pelo tamanho das perguntas, o que ocasionalmente contribuiu para a lentidão na sua execução e consequentemente travamento do sistema e dificuldades de execução. Os gestores por sua vez, salientaram que a demora na construção do simulador quase inviabilizou a realização do mesmo antes do lançamento da nova coleção, algumas informações ficaram obsoletas devido à demora para o lançamento. Convém destacar que, os gestores também criticaram o fato de que alguns vendedores tiveram acesso ao simulador antes de ter concluído o treinamento online, com efeito, suas respostas não foram corretas no simulador pela falta de conhecimento dos conteúdos da nova coleção.

A análise dos dados coletados no primeiro ciclo contribuiu para a realização de um conjunto de ações para o ciclo seguinte:

a) Implementar reconhecimento do desempenho das lojas através de um ranking, para destacar o desempenho das 10 (dez) melhores lojas.

b) Criar uma campanha de engajamento dos supervisores de região para que possam trocar de experiências sobre boas práticas de engajamento com os gerentes de lojas. 
c) Revisar o conteúdo do simulador antes de disponibiliza-lo, garantindo que o treinamento ocorra na mesma velocidade das mudanças.

d) Disponibilizar um especialista da área de vendas para o período de elaboração das questões do simulador, para garantir mais agilidade na sua construção.

e) Criar um mecanismo de pré-requisito em que, os vendedores tenham que concluir primeiro o treinamento online para depois fazer o simulador de vendas.

f) Reduzir o tamanho das questões e quantidade para que o treinamento não fique dispendioso e pesado demais para os participantes.

Da mesma forma, o segundo ciclo de aplicação do Simulador de Vendas ocorreu no período de 30 (trinta) dias no mês de outubro de 2015, buscando obter a participação de mais de 17.000 vendedores. Com os resultados da segunda aplicação, constataram-se oportunidades de melhorias e aprendizados relacionadas: a estrutura do game, engajamento dos vendedores, reconhecimento do desempenho e aprimoramento dos conteúdos do treinamento.

Estruturalmente, problemas com relação a execução do simulador de vendas nas lojas foram identificados apenas em duas regiões distintas. Tendo em vista que apenas duas regiões reclamaram, entende-se que, tais lojas estão em regiões menos privilegiadas com relação ao acesso à internet. Eventualmente, esses problemas afluem com uma determinada ênfase, mas a empresa não consegue ter gerência direta sobre esse fato. A empresa buscou assegurar a inclusão de todas as lojas e entendeu a necessidade de investir para identificar alternativas para viabilizar a execução do simulador.

Quanto ao engajamento, a Sapatus envolveu 60\% dos vendedores e atingiu a participação de $40 \%$ das lojas. Na ótica dos gestores, atribui-se esse aumento do engajamento e participação a adoção de mecanismos de reconhecimentos através do ranking das lojas. A novidade dessa proposta, trouxe uma motivação para a participação das lojas, no entanto, os vendedores destacaram que apreciariam receber um destaque pelo seu desempenho individual e citaram ações que são realizadas internamente por alguns gestores de lojas. Apesar do simulador antecipar situações de venda das novas coleções que ainda não chegaram nas lojas, as decisões dos vendedores nos contextos que foram simulados, refletiram uma oportunidade de aprimoramento dos conteúdos do treinamento. Os resultados mostraram que, os conteúdos não seriam suficientes para pré-fixar a maneira como a venda deveria ser executada, logo, uma revisão seria necessária e um complemento desenvolvido para ser melhor compreendido o conteúdo pelos intérpretes.
As ações tomadas pela empresa após a análise dos dados coletados no segundo ciclo contribuíram para melhorias nas ações no ciclo seguinte:

a) Avaliar a estrutura das lojas que estão situadas em regiões com acesso limitado a internet.

b) Estimular a troca de conhecimentos e boas práticas entre os supervisores de regiões e gerentes de lojas.

c) Adotar mecanismos de reconhecimento individual e não apenas coletivo.

d) Reforçar o treinamento online após a execução do simulador, desenvolvendo conteúdos mais aprimorados com relação a "conhecimento sobre o produto", "contorno de objeções" e "fechamento da venda".

Por sua vez, o terceiro ciclo de aplicação do Simulador de Vendas ocorreu durante o mês de outubro de 2016. Os resultados da terceira aplicação do game, indicaram oportunidades de melhorias e aprendizados relevantes, relacionadas: troca de conhecimentos e experiências entre os supervisores de região e gerentes de lojas e estratégias de engajamento dos vendedores e das lojas.

Um movimento relevante dos Supervisores de região através da criação de uma comunidade no ambiente virtual chamada de "Master Vendas" - oportunizou a socialização de "receitas de sucesso" e de "boas práticas de engajamento". Consequentemente, a consciência dos gestores sobre a importância de engajar seus times nos treinamentos foi sendo fortalecida e as práticas disseminadas para todas as lojas. Como resultado, pode se aferir o aumento da satisfação nos treinamentos de $85 \%$ para $90 \%$ de um ciclo para outro, bem como, a mobilização de $80 \%$ das lojas e o aumento da participação dos vendedores em $80 \%$. Sob o ponto de vista dos gestores, a adesão das lojas bem como o engajamento dos vendedores aumentou em função da Sapatus ter destacado os 10 (dez) vendedores com melhor desempenho e ao lado o nome da loja em que trabalhavam. À luz dessa concepção de competição, houve outro movimento dos Supervisores de região, eles criaram uma comunidade no ambiente virtual chamada de "Show de Vendas", o objetivo era divulgar fotos das lojas, mostrando seus vendedores com certificados nas mãos no momento em que todos concluíssem o treinamento. Do ponto de vista da expectativa dos gestores, a gamificação pode contribuir para avaliar o processo de aprendizagem e aumentar a eficiência dos treinamentos, contudo, reside um desafio para o próximo ciclo, buscar pela concretização destes objetivos e mudar a percepção com relação aos games até então aplicados.

As ações tomadas pela Sapatus após a análise dos dados coletados no terceiro ciclo contribuíram para melhorias nas ações no ciclo seguinte: 
a) Analisar o processo de aprendizagem e identificar o nível de prontidão geral e individual da força de vendas.

b) Predição e mitigação de problemas futuros.

Por fim, o quarto e último ciclo de aplicação do Simulador de Vendas ocorreu durante o mês de outubro de 2017 e obteve a adesão de $100 \%$ das lojas e dos vendedores. No que tange à aplicação do Game 4, delineadas as contribuições a despeito dos resultados obtidos com o simulador, cabe destacar um avanço percebido através do uso da Gamificação.

Tal avanço, proporciona uma evolução com relação a avaliação dos resultados dos treinamentos de vendas, promovendo: avaliação da eficácia do treinamento, avaliação do nível de prontidão da força de vendas e mitigação de problemas futuros. Como exemplo dessa situação, citase uma determinada etapa do Game 4 em que houve uma simulação de uma cliente informando que precisava de um sapato para ir em um casamento (era esperado que o vendedor após analisar o contexto descrito no game e o perfil da cliente) tomasse uma ação de escolher o sapato da nova coleção mais adequado para aquela oportunidade de venda. Neste caso específico, constatou-se que $95 \%$ (noventa e cinco por cento) das lojas erraram a escolha do sapato conforme a oportunidade de venda e perfil da cliente representada pelo simulador.

Os resultados indicaram que o uso da Gamificação contribui para reduzir problemas futuros com meses de antecedência da chegada dos novos produtos nos pontos de venda impactando principalmente no discurso de vendas, no conhecimento sobre as características dos produtos e principalmente nos indicadores de fechamento de vendas. A empresa atribuiu como fator de sucesso para a execução do Game 4 três variáveis principais, precedidas pela experiência adquirida nos ciclos de aprendizagem anteriores: a definição adequada do conteúdo do treinamento, a análise do processo de aprendizagem e a simulação da realidade de loja. Contudo, a disponibilidade de tempo de um especialista para o planejamento e construção dos conteúdos do simulador, ainda representa um desafio para a Sapatus. O nível de complexidade desta construção, requer a disponibilidade de tempo de um especialista da área de vendas para elaborar o conteúdo, elaborar as situações do simulador e testar a sua funcionalidade. Cabe salientar que, a Sapatus desenvolve todo o seu conteúdo e realiza a testagem com alguns profissionais de vendas para avaliar a linguagem, design de interação do game e a aderência do conteúdo a realidade das lojas.

\section{Discussão e implicações}

A análise dos resultados advindos da aplicação da gamificação nos treinamentos de vendedores da empresa
Sapatus, permitiu responder lacunas sobre como a gamificação aplicada ao treinamento de vendedores pode gerar melhores resultados para o negócio (Raybourn, 2014; Allal-Chérif; Makhlouf, 2016) e quais são as melhores práticas para o treinamento desses profissionais ( $\mathrm{Li}$; JhangLi, 2010; Raybourn, 2014).

Os achados da pesquisa sugerem que a Gamificação aprimorou o desempenho dos vendedores nas seguintes principais dimensões: (i) argumentos de vendas, (ii) conhecimento dos produtos, e (iii) alternativas para o fechamento da venda. A gamificação também indicou oportunidades para o aprimoramento de conteúdo dos treinamentos, análise do processo de aprendizagem, e para a mudança de percepção sobre os games.

A escolha pela pesquisa-ação como metodologia, através da experiência adquirida a cada ciclos de melhoria e aprendizagem, provou aumentar a eficiência geral dos treinamentos, contribuindo para: avaliar os resultados dos treinamentos de vendas; avaliar do nível de prontidão dos vendedores e desvelou uma postura que amplia a utilização da Gamificação como estratégia para mitigar problemas que ainda não ocorreram.

\subsection{Impactos da gamificação nos resultados de treinamentos de vendas}

Os achados indicam que a gamificação foi responsável por gerar maior engajamento e participação dos vendedores nos treinamentos de vendas da empresa Sapatus, aumentando na ordem de $20 \%$ para $100 \%$. Esse resultado foi alcançado em razão do investimento da Sapatus para tornar os conteúdos dos treinamentos mais atrativos (Raybourn, 2014), adicionando elementos motivacionais por meio da Gamificação (Domínguez et al., 2013; Jung; Kim; Lee, 2014; Mačiuliené; Skaržauskienè, 2016), com efeito, obtevese maior interesse dos vendedores nos treinamentos. (AllalChérif; Makhlouf, 2016).

Os achados da pesquisa mostraram que a gamificação gerou maior mobilização das lojas nos treinamentos, aumentando a participação de $20 \%$ para $100 \%$ entre o primeiro e quarto ciclo. Atribui-se esse resultado a adoção da competição embasadas por objetivos de desempenhos individuais e coletivos (Mačiuliene; Skaržauskiene, 2016). A Sapatus inicialmente adotou um ranking para reconhecer as lojas, mas apenas alcançou a mobilização de todas quando incluiu o ranking que destacava os 10 melhores desempenhos dos vendedores associados à sua loja de origem.

Resultados verificados também incluíram o aumento da satisfação dos treinandos com relação aos treinamentos de vendas numa ordem de $70 \%$ para $95 \%$. A literatura apresenta os benefícios potenciais advindos do uso da gamificação em programas de treinamento de vendas para promover a qualidade dos programas de aprendizagem (Qudrat- 
Ullah, 2010; Gordon et al., 2012; Howard, 2017) e alinhar as estratégias de formação de vendedores aos objetivos organizacionais na área de vendas (Moncrief; Marshall; Rudd, 2015; Singh; Venugopal, 2015).

\subsection{Impacto da gamificação no nível de prontidão dos vendedores}

Os resultados deste estudo adicionam a importância de considerar que o uso de simuladores fornece a oportunidade de identificar as ações dos participantes para os tomadores de decisões, como também é uma ferramenta útil de feedback aos próprios usuários, que poderão testar suas decisão em ambientes controlados e seguros antes que a implementação real (Tang; Austin, 2009). Em termos de avaliação da aprendizagem, resultados deste estudo mostraram que nas simulações, as percepções de realismo são criadas através do ambiente e do controle das interações. Com efeito, é possível avaliar como os usuários se portaram em situações de tomada de decisões (Allal-Chérif; Makhlouf, 2016).

A aplicação da gamificação através de um game de simulação, permitiu a Sapatus avaliar competências específicas dos vendedores e estabelecer quais habilidades não haviam sido adquiridas. A partir da estrutura criada para a medição do conjunto de dados relativos às atribuições dos vendedores, foi possível avaliar e comparar o desempenho de cada vendedor para a aplicação do conhecimento (DeMarcos; Garcia-Lopez; Garcia-Cabot, 2016). A literatura denomina esse acompanhamento de "fluxo de absorção do conhecimento", que ocorre através da análise do nível de apropriação da competência explicitada na sua tomada de decisão (De-Marcos et al., 2017). O fluxo de conhecimento nos treinamentos de vendas da Sapatus, apresentou o registro de todas as ações dos usuários através do seu progresso dentro do game, mostrando ao final se a eficácia do aprendizado foi alcançada (Westera, 2017).

Os resultados indicam que esse monitoramento pode ajudar a antecipar ações que atenuem a postura de resistência a participar de programas de treinamento. (Andrade; Mizoguchi; Isotani, 2016). A composição da simulação do ambiente loja da Sapatus apresentou situações e contextos controlados para testar a capacidade dos vendedores em análise de situações (Yalabik; Howard; Roden, 2012), tomada de decisões (Bevilacqua et al., 2015), administração de imprevistos e eventos inesperados, otimização de recursos e coordenação de habilidades (Singh; Venugopal, 2015; Howard, 2017; Westera, 2017), concentrar esforços da área comercial em diagnóstico e treinamento (Singh; Venugopal, 2015; Allal-Chérif; Makhlouf, 2016), aprimorar o desempenho profissional (Jung; Kim; Lee, 2014; Singh; Venugopal, 2015; Allal-Chérif; Makhlouf, 2016), descrever as interações do jogador, navegação e história de atividades e conquistas (Serrano-Laguna et al., 2017; Westera, 2017), registrar e acompanhar os estágios iniciais e de desenvolvimento do usuário (Serrano-Laguna et al., 2017; Westera, 2017), investigar profundamente as variáveis e tomadas de decisões (Singh; Venugopal, 2015; SerranoLaguna et al., 2017; Westera, 2017) e permitir feedback imediato, (Gordon et al., 2012; Yalabik; Howard; Roden, 2012).

O estudo mostrou três pontos importantes que precisam ser observados com relação a adoção de simuladores para treinamentos de vendas. Primeiro, para que se tenha uma adequada execução do simulador de vendas, é imprescindível que os vendedores obtenham um conhecimento prévio sobre os produtos e processos da área onde trabalham. O que não ocorreu no primeiro ciclo deste estudo, onde foi aplicado o primeiro game de simulação.

O estudo mostrou a importância de criar mecanismos que obrigassem os vendedores passarem primeiro pelo treinamento online e depois chegarem ao simulador. Segundo, resultados adicionam que, o desenvolvimento de games simuladores para a aprendizagem na área de vendas não podem ser muito complexos e dispendiosos (Boyle et al., 2016), pois poderão afetar a eficácia da aprendizagem, considerando, por exemplo, as motivações dos vendedores (Westera, 2017) e até mesmo sua habilidade para o uso de tecnologias (Robinson; Marshall; Stamps, 2005). Esse cuidado pode ajudar a eliminar algumas barreiras como apreensão de alguns profissionais para o uso da tecnologia (Hamari; Keronen, 2017). Terceiro, o estudo mostrou que o reconhecimento baseado no ranking que expunha os melhores desempenhos das lojas foi menos eficiente do que o reconhecimento individual de cada vendedor (Lee; Ahn, 2007).

Por fim, os resultados a partir de cada game permitiram a empresa corrigir e aplicar melhorias em processos de forma ágil. Resultados mostram que a aplicação do simulador ajudou na identificação de pontos fracos e falhas na execução de procedimentos da área de vendas pelos vendedores. Isso permitiu que a Sapatus realizasse ações preventivas sobre as causas, gerando oportunidades de mudanças mais significativas nos processos a partir da análise dos resultados de cada game simulador. A gamificação se tornou preditiva, pois, criou contextos simulados e controlados, onde os registros de dados sobre a tomada de decisão e os caminhos percorridos pelos vendedores, buscaram predizer comportamentos futuros.

\section{Conclusões}

O presente estudo revelou que o uso da Gamificação no Treinamento da força de vendas de um fabricante global de calçados, que opera em mais de 18 países, possibilitou avaliar o nível de prontidão da força de vendas e, mitigar problemas futuros com dois meses de antecedência da chegada dos novos produtos em Lojas.

$\mathrm{Na}$ fase de análise do processo de aprendizagem, dois importantes elementos foram destacados para a 
simulação: ambiente e controle. Em termos de avaliação da aprendizagem, resultados deste estudo mostraram que é possível avaliar competências específicas dos vendedores e identificar quais habilidades não haviam sido adquiridas através do mapeamento do fluxo de conhecimento. As alternativas para mitigar problemas nesta etapa de execução incluem, a necessidade de escolher estratégias avaliativas de forma que estejam alinhadas com os objetivos do treinamento e ainda, realizar um monitoramento constante a partir das interações dos vendedores.

$\mathrm{Na}$ fase de simulação de loja, a Sapatus apresenta a contribuição da gamificação para registrar e acompanhar os estágios iniciais e de desenvolvimento do usuário, mapeando as variáveis e tomadas de decisões no game simulador. Nesta etapa, o uso da gamificação, aumentou os níveis de conhecimentos dos vendedores, aumentou suas habilidades, aprimorou a capacidade de vender e aumentou a participação inclusive dos funcionários geograficamente dispersos.

Os resultados deste artigo evidenciam uma ampliação do uso da gamificação que contribui para predizer e mitigar problemas futuros. Contudo, as alternativas para mitigar problemas nesta etapa de execução incluem, cuidados com o desenvolvimento de games simuladores para que não sejam muito complexos e dispendiosos e avaliar as possíveis barreiras oriundas da resistência de alguns profissionais para o uso da tecnologia.

Como em qualquer estudo, essa pesquisa tem limitações que devem ser abordadas. Como o estudo é baseado em um estudo de caso único, os resultados não podem ser estatisticamente generalizados para uma população de fabricantes de calçados (Eisenhardt, 1991). Além disso, a análise de um único caso de aplicação da gamificação no treinamento de vendas impede a comparação direta com outros casos. Este estudo foi realizado no Brasil, o que significa que os resultados devem considerar as características dos países em desenvolvimento em comparação com os países industrializados (Schoenherr et al., 2012). Estudos futuros podem investigar como a gamificação pode projetar novos produtos ou serviços inovadores através do treinamento na área de vendas? Ou ainda, como alavancar resultados financeiros de loja a partir do uso da Gamificação em treinamentos de vendas?

\section{Referências}

Albers, S., Raman, K. and Lee, N. (2015), "Trends in optimization models of sales force management", Journal of Personal Selling and Sales Management, Vol. 35 No. 4, pp. 275-291.

Allal-Chérif, O. and Makhlouf, M. (2016), "Using serious games to manage knowledge: The SECI model perspective", Journal of Business Research, Elsevier Inc., Vol. 69 No. 5, pp. 1539-1543.
Andrade, F.R.H., Mizoguchi, R. and Isotani, S. (2016), "The bright and dark sides of gamification", Lecture Notes in Computer Science (Including Subseries Lecture Notes in Artificial Intelligence and Lecture Notes in Bioinformatics), Vol. 9684, pp. 176-186.

Attali, Y. and Arieli-Attali, M. (2015), "Gamification in assessment: Do points affect test performance?", Computers and Education, Elsevier Ltd, Vol. 83, pp. 57-63.

Bachrach, D.G., Mullins, R.R. and Rapp, A.A. (2017), "Intangible sales team resources: Investing in team social capital and transactive memory for marketdriven behaviors, norms and performance", Industrial Marketing Management, Elsevier Inc., Vol. 62, pp. 8899.

Bashaw, R.E., Ingram, T.N. and Keillor, B.D. (2002), "Improving sales training cycle times for new trainees. An exploratory study", Industrial Marketing Management, Vol. 31 No. 4, pp. 329-338.

Buckley, P. and Doyle, E. (2017), "Individualising gamification: An investigation of the impact of learning styles and personality traits on the efficacy of gamification using a prediction market", Computers and Education, Vol. 106, pp. 43-55.

Chonko, L.B., Dubinsky, A.J., Jones, E. and Roberts, J.A. (2003), "Organizational and individual learning in the sales force: An agenda for sales research", Journal of Business Research, Vol. 56 No. 12, pp. 935-946.

De-Marcos, L., Garcia-Lopez, E. and Garcia-Cabot, A. (2016), "On the effectiveness of game-like and social approaches in learning: Comparing educational gaming, gamification \& social networking", Computers and Education, Vol. 95, pp. 99-113.

De-Marcos, L., Garcia-Lopez, E., Garcia-Cabot, A., Connolly, T.M., Boyle, E.A., Macarthur, E., Hainey, T., et al. (2017), "Perceptions of the effectiveness of system dynamics-based interactive learning environments: An empirical study", Computers and Education, Elsevier Ltd, Vol. 37 No. 3, pp. 1529-1533.

De Freitas, S. and Ketelhut, D.J. (2014), "Introduction for the Journal of Information Sciences special issue on serious games", Information Sciences, Vol. 264, pp. 1-3.

Gordon, G.L., Shepherd, C.D., Lambert, B., Ridnour, R.E. and Weilbaker, D.C. (2012), "The training of sales managers: current practices", Journal of Business and Industrial Marketing, Vol. 27 No. 8, pp. 659-672. 
Groza, M.D., Locander, D.A. and Howlett, C.H. (2016), "Linking thinking styles to sales performance: The importance of creativity and subjective knowledge", Journal of Business Research, Vol. 69 No. 10, pp. 41854193.

Halkett, R. (2002), "E-learning and how to survive it", Industrial and Commercial Training, Vol. 34 No. 2, pp. 80-82.

Hamari, J. and Koivisto, J. (2015), "Why do people use gamification services?", International Journal of Information Management, Elsevier Ltd, Vol. 35 No. 4, pp. $419-431$.

Honeycutt, E.D., Karande, K.W. and Jantan, M.A. (2002), "Sales training in Malaysia. High- vs. Low-tech methods", Industrial Marketing Management, Vol. 31 No. 7, pp. 581-587.

Jones, E., Chonko, L.B. and Roberts, J.A. (2004), "Sales force obsolescence: Perceptions from sales and marketing executives of individual, organizational, and environmental factors", Industrial Marketing Management, Vol. 33 No. 5, pp. 439-456.

Jr, J.F.T., Fournier, C., Wise, J.A., Hollet, S. and Poujol, J. (2008), "Executives' perspectives of the changing role of the sales profession: views from France, the United States, and Mexico", Journal of Business and Industrial Marketing, Vol. 23, pp. 193-202.

Jung, H.S., Kim, K.H. and Lee, C.H. (2014), "Influences of perceived product innovation upon usage behavior for MMORPG: Product capability, technology capability, and user centered design", Journal of Business Research, Elsevier Inc., Vol. 67 No. 10, pp. 2171-2178.

Kananen, J. (2013), "Gamification of the sales process at a telecommunications company to improve the motivation of the salesforce".

Kapp, K. (2012), "The Gamification of Learning and Instruction, Pfeiffer", San Francisco, p. 480.

Lauto, G. and Valentin, F. (2016), "How preference markets assist new product idea screening.", Industrial Management and Data Systems, Vol. 116 No. 3, pp. 603-619.

Li, Y.-M. and Jhang-Li, J.-H. (2010), "Knowledge sharing in communities of practice: A game theoretic analysis", European Journal of Operational Research, Elsevier B.V., Vol. 207 No. 2, pp. 1052-1064.
Mačiuliene, M. and Skaržauskiene, A. (2016), "Evaluation of co-creation perspective in networked collaboration platforms", Journal of Business Research, available at: https://doi.org/10.1016/j.jbusres.2016.04.038

Malegiannaki, I. and Daradoumis, T. (2017), "Analyzing the educational design, use and effect of spatial games for cultural heritage: A literature review", Computers and Education, Vol. 108, pp. 1-10.

Marshall, G.W., Goebel, D.J. and Moncrief, W.C. (2003), "Hiring for success at the buyer-seller interface", Journal of Business Research, Vol. 56 No. 4, pp. 247-255.

Murovec, N. and Prodan, I. (2009), “Absorptive capacity, its determinants, and influence on innovation output: Cross-cultural validation of the structural model", Technovation, Elsevier, Vol. 29 No. 12, pp. 859-872.

Murphy, W.H. and Dacin, P.A. (2009), "Sales contest research: Business and individual difference factors affecting intentions to pursue contest goals", Industrial Marketing Management, Elsevier Inc., Vol. 38 No. 1, pp. 109-118.

Murphy, W.H., Dacin, P.A. and Ford, N.M. (2004), "Sales Contest Effectiveness: An Examination of Sales Contest Design Preferences of Field Sales Forces", Journal of the Academy of Marketing Science, Vol. 32 No. 2, pp. 127-143.

Rapp, A., Marcengo, A., Console, L. and Simeoni, R. (2012), "Playing in the wild", Proceeding of the 16th International Academic MindTrek Conference on MindTrek '12, No. August 2016, p. 227.

Raybourn, E.M. (2014), “A new paradigm for serious games: Transmedia learning for more effective training and education", Journal of Computational Science, Elsevier B.V., Vol. 5 No. 3, pp. 471-481.

Ricks, J.M., Williams, J.A. and Weeks, W.A. (2008), "Sales trainer roles, competencies, skills, and behaviors: A case study", Industrial Marketing Management, Vol. 37 No. 5, pp. 593-609.

Robson, K., Plangger, K., Kietzmann, J.H., McCarthy, I. and Pitt, L. (2015), "Is it all a game? Understanding the principles of gamification", Business Horizons, "Kelley School of Business, Indiana University”, Vol. 58 No. 4, pp. 411-420. 
Rodriguez, M., Honeycutt, E.D. and Ragland, C. (2015), "Preliminary Investigation of Entertainment Strategies Involving Alcohol: Implications for Professional Sales Education and Training in Business Markets.”, Journal of Business-to-Business Marketing, Vol. 22 No. 4, pp. 257-268.

Schillewaert, N., Ahearne, M.J., Frambach, R.T. and Moenaert, R.K. (2005), "The adoption of information technology in the sales force", Industrial Marketing Management, Vol. 34 No. 4 SPEC ISS., pp. 323-336.

Senecal, S., Pullins, E.B. and Buehrer, R.E. (2007), "The extent of technology usage and salespeople: an exploratory investigation", Journal of Business and Industrial Marketing, Vol. 22 No. 1, pp. 52-61.

Serna, E., Bachiller, O. and Serna, A. (2017), "Knowledge meaning and management in requirements engineering", International Journal of Information Management, Elsevier Ltd, Vol. 37 No. 3, pp. 155-161.

Serrano-Laguna, Á. and Fernández-Manjón, B. (2014), "Applying learning analytics to simplify serious games deployment in the classroom", IEEE Global Engineering Education Conference, EDUCON, pp. 872-877.
Singh, R. and Venugopal, P. (2015), "The impact of salesperson customer orientation on sales performance via mediating mechanism", Journal of Business and Industrial Marketing, Vol. 30 No. 5, pp. 594-607.

Singh, V.L., Manrai, A.K. and Manrai, L.A. (2015), "Sales training: A state of the art and contemporary review", Journal of Economics, Finance and Administrative Science, AEDV, Vol. 20 No. 38, pp. 54-71.

Tang, T.L.-P. and Austin, M.J. (2009), “Students' perceptions of teaching technologies, application of technologies, and academic performance", Computers and Education, Vol. 53 No. 4, pp. 1241-1255.

Wall, J. and Ahmed, V. (2008), "Use of a simulation game in delivering blended lifelong learning in the construction industry - Opportunities and Challenges", Computers and Education, Vol. 50 No. 4, pp. 1383-1393.

Weinrauch, J.D., Stephens-friesen, M. and Carlson, R.L. (2001), "Improving the viability of manufacturers ' representatives with industry-based sales training initiatives", Journal of Business and Industrial Marketing, Vol. 16 No. 3, pp. 183-196. 